\title{
Análise da superfície foliar e agrupamento de 10 espécies arbóreas: uma ferramenta na identificação de espécies Amazônicas
}

Analysis of leaf surface and clustering of $\mathbf{1 0}$ tree species: a tool in the identification of Amazonian species

Análisis de superficie foliar y agrupamiento de 10 especies arbóreas: una herramienta en la identificación de especies amazónicas

\section{Resumo}

Objetivou-se descrever e sistematizar a epiderme foliar de 10 espécies florestais amazônicas, com ferramentas auxiliar a taxonomia, contribuindo com o conhecimento técnico-científico. Para isso realizou-se técnicas usuais em anatomia vegetal, para observação, além da largura, comprimento e frequência dos estômatos e submetidos a estatística, aplicando: o teste de Tukey, ANOVA, Análise de agrupamento e Análise de Componentes Principais. Observou-se que a epiderme adaxial e abaxial as células são heterodimencionais, com forma e tamanho variados, arredondadas e sinuosas, em todas as espécies os estômatos são anomocíticos e somente a Spondias mombin é anfiestomatica, as espécies Dipteryx odorata e Virola surinamensis podem ser facilmente identificadas devido sua ornamentação da célula guarda, as espécies Alexa grandiflora e Lecythis lurida apresentam estruturas semelhantes dificultando a identificação. Os tricomas apresentaram formas variadas, sendo estes o melhor parâmetro para identificação e distinção. As Fabaceae são as que possuem maior diversidade de estrutura dificultando o agrupamento, a frequência estomática pode ser utilizada com sucesso para agrupamento das espécies estudadas.

Palavras-chave: Anatomia vegetal; Estruturas anatomia; Identificação foliar, Epiderme.

\begin{abstract}
The objective was to describe and systematize the leaf epidermis of 10 Amazonian forest species, with tools to help the taxonomy, contributing with the technical-scientific knowledge. For this, the usual techniques in plant anatomy were performed, for observation, besides the stomata width, length and frequency and submitted to statistics, applying: Tukey test, ANOVA, Cluster Analysis and Principal Component Analysis. The adaxial and abaxial epidermis cells are heterodimensional, with varying shape and size, rounded and sinuous, in all species the stomata are anomocytic and only Spondias mombin is amphiestomatic, Dipteryx odorata and Virola surinamensis species can be easily Identified due to their guard cell ornamentation, the species Alexa grandiflora and Lecythis lurida have similar structures making identification difficult. The trichomes presented varied forms, being these the best parameter for identification and distinction. The Fabaceae are the ones that have greater structure diversity, making the grouping difficult, the stomatal frequency can be successfully used to group the studied species.
\end{abstract}

Keywords: Plant anatomy; Anatomical structures; Leaf identification; Epidermis.

\section{Resumen}

El objetivo fue describir y sistematizar la epidermis foliar de 10 especies del bosque amazónico, con herramientas de apoyo a la taxonomía, aportando conocimientos técnicos y científicos. Para ello, se realizaron técnicas habituales en anatomía vegetal, para la observación, además del ancho, largo y frecuencia de los estomas y sometidas a estadística, aplicando: la prueba de Tukey, ANOVA, análisis de conglomerados y análisis de componentes principales. Se observó que la epidermis adaxial y abaxial las células son heterodimensionales, de variada forma y tamaño, 
redondeadas y sinuosas, en todas las especies los estomas son anomocíticos y solo Spondias mombin es anfhistomático, las especies Dipteryx odorata y Virola surinamensis pueden identificarse fácilmente debido a Debido a la ornamentación de la celda de guardia, las especies Alexa grandiflora y Lecythis lurida presentan estructuras similares, lo que dificulta la identificación. Los tricomas presentaron formas variadas, que son el mejor parámetro para la identificación y distinción. Las fabáceas son las que tienen mayor diversidad de estructura, lo que dificulta la agrupación, la frecuencia estomática puede utilizarse con éxito para agrupar las especies estudiadas.

Palabras clave: Anatomía vegetal; Estructuras anatómicas; Identificación foliar; Epidermis.

\section{Introdução}

O conhecimento técnico-cientifico sobre as diversas partes constituintes do vegetal fornecem informações importantes sobre as variações inter e intraespecífica, bem como as suas estratégias adaptativas. Neste sentido, a folha é um dos órgãos vegetais que possui maior plasticidade morfo-anatômica, embora seja amplamente utilizada na identificação de espécies.

Com isso, vários caracteres foliares foram e ainda são utilizados com sucesso na taxonomia e sistematização dos vegetais e representam um significativo avanço na ciência das plantas, com a criação de sistemas de classificação complexos, completos e de fácil manuseio, para a época em que foram criados, destacando-se: o de Cronquist e Engler.

A medida em que a tecnologia avança, novas técnicas e procedimentos são adicionados o que detalha cada vez mais as estruturas vegetais e melhoram a compreensão, distinção e estratégias adaptativas desses vegetais. E com isso, cada vez mais, pesquisadores procuram maneiras de facilitar e baratear a identificação e sistematização das plantas.

Uma questão problemática na região amazônica é a troca involuntária e/ou dolosa de espécies nos inventários florestais e estes equívocos trazem consequências e imagens negativas sobre a atividade manejo florestal na região e consequentemente prejuízos ao comercio de produtos de base florestal. Essas trocas são ocasionadas pela semelhança morfológica entre as espécies, pois em muitos casos a identificação é realizada somente pelos caracteres vegetativos e a folha é a principal parte utilizada.

Outro fator é o uso de nomes vernaculares que segundo Oliveira (2011), do ponto de vista comercial, a prática de nomear espécies por nomes vernaculares pode ser prejudicial, tanto ao consumidor quanto ao empresário, principalmente pela falta da garantia da qualidade do produto comercializado. E variação de nomes é afetada pelos dialetos locais e caraterísticas das espécies, que fazem parte do conhecimento popular local. (Cysneiros et al., 2018).

Como mencionado, a folha é a parte da planta mais utilizada na identificação em campo e com isso, trabalhos que enfoquem a descrição e/ou detalhamento desta parte e que adicionem modelagem matemáticas e estatísticas tornam-se uteis e facilitam a identificação. Ressalta-se que, atualmente, técnicas básicas aliadas a modelagens matemáticas e inteligência artificial estão se tornando ferramentas importantes para classificações precisas, rápidas e eficazes nos agrupamentos e estão sendo utilizadas em diversas áreas do conhecimento

As características da epiderme foliar foram e ainda são utilizadas na taxonomia e classificação de alguns táxons conforme realizado nos trabalhos por Kong (2001); Akcin et al, (2013); Klimko et al., (2018); Rashid (2019); Gul et al (2019a,b); Tomaszewski et al (2019) e Nazish (2020), porém essas pesquisas não utilizaram somente a descrição qualitativa, incluíram análises quantitativas e estas responderam muito bem na classificação.

Outra vertente, atualmente, são os trabalhos que utilizam as características e/ou imagens aliado a modelagem matemática e inteligência artificial já são reportadas na literatura como os trabalhos de Silva et al. (2016) e Mele e Gargiulo (2020) que utilizaram imagens da epiderme para identificação e classificação de espécies vegetais.

Diante do exposto, o presente trabalho tem como objetivo descrever, caracterizar quimicamente estruturas desconhecidas a por meio de espectroscopia de energia dispersiva, e sistematizar, através de análises de agrupamento, a epiderme foliar e de folíolos de dez espécies florestais amazônicas, contribuindo com o conhecimento técnico-científico de espécies arbóreas e como ferramentas auxiliar a taxonomia. 


\section{Metodologia}

\section{Material Botânico}

As amostras foram obtidas através de exsicatas disponibilizadas pelo acervo do Centro de Estudos Ambientais (CEA), centro criado pela empresa que construiu a hidroelétrica de belo monte para resgate e monitoramento da fauna e flora e a coleta foi realizada na área de monitoramento da norte energia e área influenciada diretamente pela construção da Usina Hidrelétrica de Belo Monte, localizada no município de Vitória do Xingu, Pará, Brasil (Figura 1).

Figura 1. Mapa de localização das áreas de coleta.

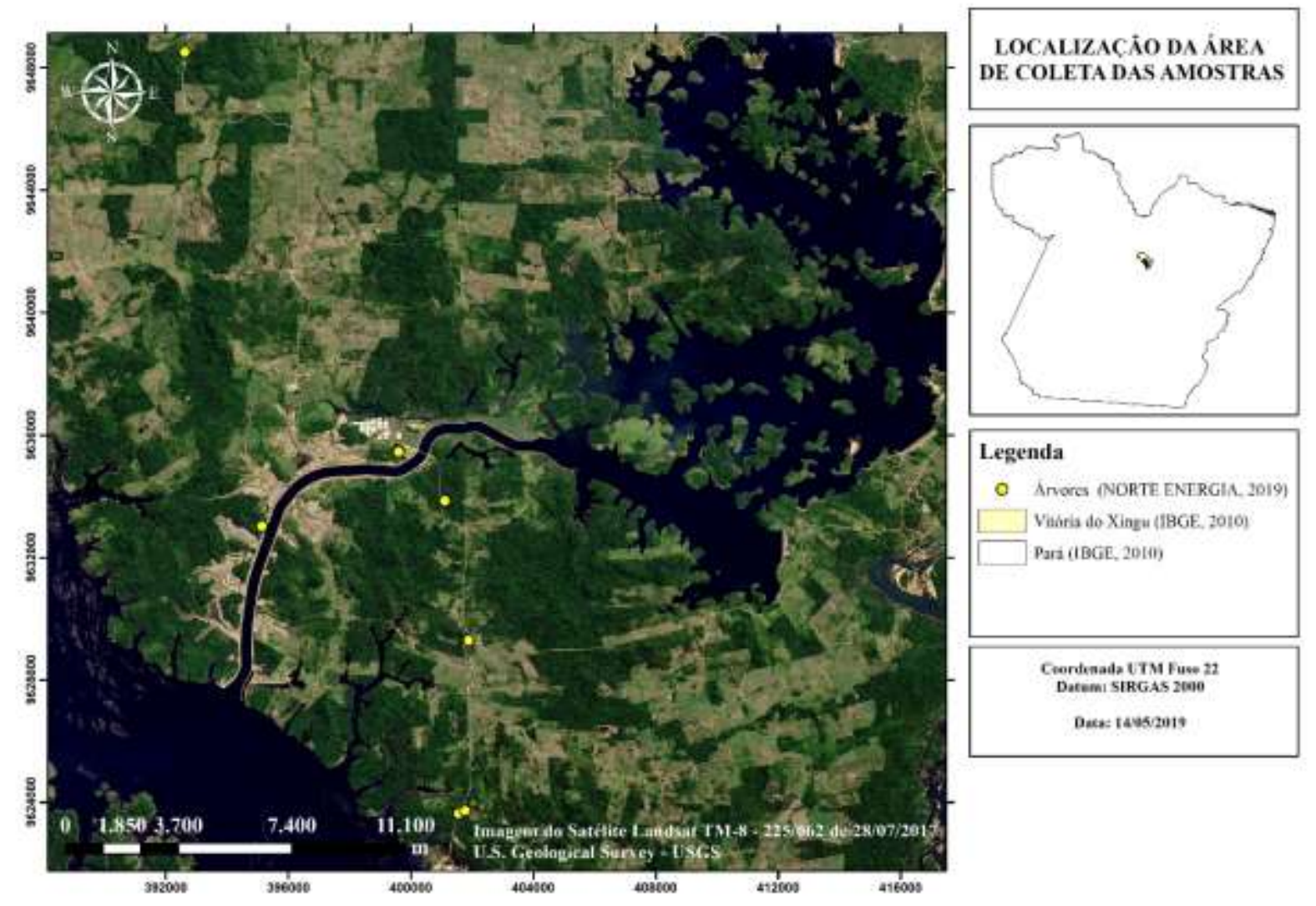

Fonte: Autores.

As dez espécies amazônicas estudadas tiveram como critério de seleção o material botânico devidamente identificado e depositado em herbário, os quais foram reunidos pelo CEA no período entre 19 de julho de 2018 a 18 de janeiro de 2019. As plantas estudadas estão distribuídas em oito diferentes famílias botânicas (Tabela 1). 
Tabela 1. Espécies estudadas através de exsicatas doadas pelo CEA.

\begin{tabular}{|ccc|}
\hline Nome Vernacular & $\begin{array}{c}\text { Nome } \\
\text { Científico }\end{array}$ & Família \\
\hline Cajá & Spondias mombin L. & Anacardiaceae \\
\hline Cumarú & Dipteryx odorata (Aubl.) Willd. & Fabaceae \\
\hline Macharimbé & Cenostigma tocantinum Ducke. & Fabaceae \\
\hline Melancieira & Alexa grandiflora Ducke. & Fabaceae \\
\hline Lacre & Vismia guianensis (Aubl.) Choisy. & Hypericaceae \\
\hline Pau Cravo & Dicypellium caryophyllaceum & Lauraceae \\
\hline Jarana & (Mart.) Nees. & Lecythidaceae \\
\hline Mutamba & Lecythis lurida (miers) S.A.Mori. & Meliaceae \\
\hline
\end{tabular}

Fonte: Autores.

\section{Dissociação Foliar}

Para os estudos anatômicos, foram selecionadas três folhas de cada exsicata e em cada ponto de coleta (figura 1) foram coletadas, pelos menos, 3 espécimes de cada espécie, totalizando 180 amostras analisadas, tendo como critério folhas inteiras e adultas, de forma aleatória e, em seguida, cada folha foi dividida em quatro regiões sendo elas: ápice, nervura central, margem e base. As folhas foram imersas em solução de água + hipoclorito de sódio (1:1) e aquecidas em placa aquecedora a $250^{\circ} \mathrm{C}$.

Após a total dissociação da epiderme, as amostras foram lavadas em água destilada e coradas em azul de toluidina e fucsina básica (JUNQUEIRA, 1990), seguindo de bateria de série etílica e aceto-butílica para desidratação, e montadas entre lâmina e lamínula com balsamo do Canadá (KRAUS e ARDUIN, 1997). Fotomicrografias foram capturadas em microscópio biológico trinocular 1600x com câmera e software opticam 3.7, sendo as análises realizadas no laboratório de morfoanatomia vegetal e qualidade da madeira da Universidade Federal do Pará, campus Altamira.

\section{Microscopia Eletrônica de Varredura (MEV)}

Para análise da epiderme em Microscopia Eletrônica de Varredura (MEV) foram utilizadas amostras de lâminas foliares das regiões adaxial e abaxial, também provenientes de material herborizado. O material foi fixado sobre placa de vidro com o auxílio de fita dupla face, onde foi realizada a metalização de ouro (Metalizador K550X, EMITECH). Fotomicrografias foram capturadas por meio de microscópio eletrônico de varredura da marca (MEV LEO-1430, ZEISS), sendo as análises realizadas no laboratório de Microanálises do Instituto de Geociências (IG) da Universidade Federal do Pará, câmpus Belém. 


\section{Espectroscopia de Energia Dispersiva (EDS)}

As análises de Espectroscopia de Energia Dispersiva, do inglês Energy Dispersive Spectroscopy (EDS), foram realizadas no Laboratório de Microanálises do Instituto de Geociências (IG) da UFPA, campus Belém, com as mesmas amostras utilizadas para o MEV. O equipamento utilizado foi um MEV Zeiss modelo LEO-1430 com EDS IXRF modelo Sirius-SD acoplado. As condições de operação foram: corrente do feixe de elétrons $=90 \mu \mathrm{A}$, voltagem de aceleração constante $=20 \mathrm{kV}$, distância de trabalho $=15 \mathrm{~mm}$, tempo de contagem para análise dos elementos $=30 \mathrm{~s}$.

\section{Análise Estatística}

Para a contagem dos estômatos, foram selecionadas aleatoriamente dez campos em todas as folhas e folíolos de cada amostra, cada campo desse medindo $0,5 \mathrm{~mm}^{2}$. Já para a mensuração dos estômatos, em cada amostra selecionou-se cinco campos e dez estômatos, totalizando 50 amostragens em cada amostra, tendo como unidade de medida micrometro $(\mu \mathrm{m}) \mathrm{e}$ submetidos a Análise de Variância (ANOVA), onde foram calculadas média e desvio-padrão para quantidade por $\mathrm{mm}^{2}$, comprimento e largura dos estômatos e realizada comparação entre as espécies.

A análise de componentes principais (PCA) foi utilizada para determinar a maior variância entre os atributos selecionados. E na análise de cluster, gráficos do método hierárquico, descrito por Vicini e Souza (2005) como uma série de sucessivos agrupamentos ou sucessivas divisões de elementos, onde os elementos são agregados ou desagregados, sendo representados pelo diagrama bidimensional, denominado dendrograma ou diagrama em árvore, foram utilizados para criar grupos de espécies semelhantes.Com a análise de agrupamentos, de acordo com Sousa (2018), é possível classificar objetos de modo que cada objeto seja semelhante aos outros no agrupamento de acordo com o conjunto de características escolhidas, assim, a homogeneidade dos objetos dentro dos grupos e a heterogeneidade entre os demais grupos são maximizadas

Análises de agrupamento foram realizadas para verificar semelhanças e diferenças na forma de distribuição e tamanho dos estômatos das diferentes espécies e famílias botânicas e foram realizadas utilizando o programa StatGraphics Centurion versão Centurion XVI onde foi considerado $\alpha=0,05$ como estatisticamente significante em todos os testes.

\section{Resultados e Discussão}

As células da superfície adaxial da lâmina foliar possuem tamanhos e formas variadas da parede celular (Figura 2): (i) curva (e.g., Carapa guianensis); (ii) poligonal (e.g., Spondias mombin, Dipteryx odorata, Lecythis lúrida, Alexa grandiflora, Guazuma ulmifolia e Virola surinamensis); and (iii) sinuosa (e.g., Vismia guianensis, Cenostigma tocantinum e Dicypellium caryophyllaceum) Pode-se destacar que a sinuosidade da epiderme de uma folha, está diretamente ligada ao ambiente que a folha se desenvolve já que, paredes celulares com sinuosidades estão ligadas à exposição das plantas à diferentes intensidades luminosas do ambiente em que se desenvolvem, e o espessamento das paredes dessas células pode estar associado ao desenvolvimento em altos níveis de luminosidade (Araújo; Mendonça 1998; Alquini et al., 2003). Ou possibilidade para essa sinuosidade são as tensões ocorridas durante a diferenciação das células ou ao endurecimento da cutícula (Alquini et al., 2003) 
Research, Society and Development, v. 10, n. 2, e58810212961, 2021

(CC BY 4.0) | ISSN 2525-3409 | DOI: http://dx.doi.org/10.33448/rsd-v10i2.12961

Figura 2. A-J. Prancha da epiderme adaxial das espécies. A. Parede celular curva Carapa guianenses; B. Parede celular poligonal Spondias mombin; C. Parede celular poligonal Dipteryx odorata; D. Parede celular poligonal Lecythis lúrida; E. Parede celular sinuosa Vismia guianensis; F. Parede celular sinuosa Cenostigma tocantinum; G. Parede celular poligonal Alexa grandiflora; H. Parede celular poligonal Guazuma ulmifolia; I. Parede celular sinuosa Dicypellium caryophylaceum; J. Parede celular poligonal Virola surinamensis.
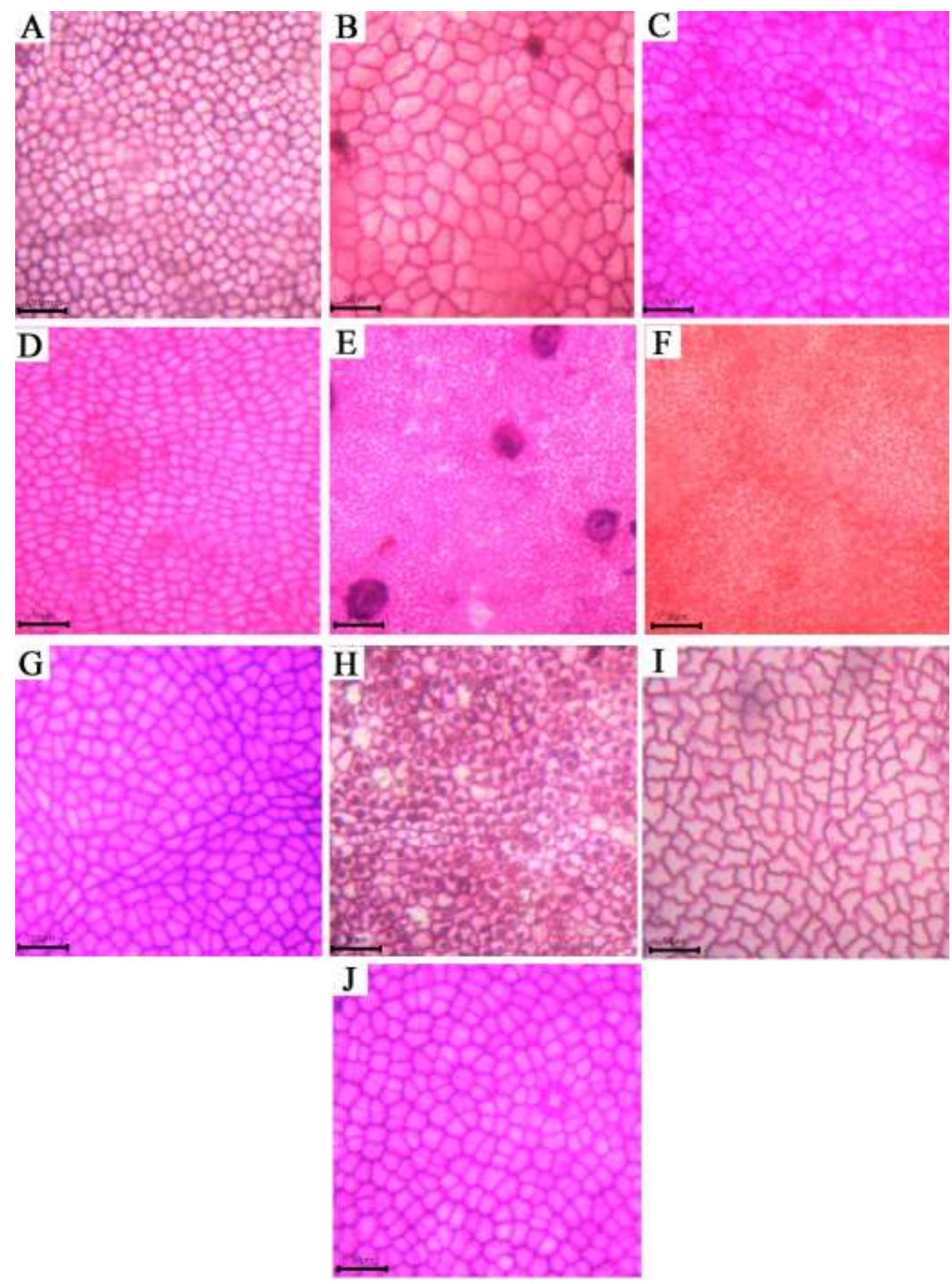

Fonte: Autores.

Todas as espécies estudadas apresentaram estômato anomocítico (Tabela 2) e com isso este parâmetro não deve ser utilizado para classificação das espécies estudadas, descrito por Metcalfe e Chalk (1950) como envolvido por um número variável de células que não diferem em formato e tamanho das demais células epidérmicas. 
Carpenter (2006) relata a importância dos tipos de complexos estomáticos na definição de caracteres homólogos para a definição de angiospemas basais. $\mathrm{O}$ autor relata ainda a dificuldade em definir a ancestralidade com base no tipo do estômato e as duas teorias envolvidas na classificação dos estômatos anomocíticos

A classificação dos estômatos tem sido realizada com sucesso para a taxonomia, distinção de taxas do mesmo grupo conforme descrito nos trabalhos Deng et al (2014) e Chen et al (2008). Nesses trabalhos, os autores encontraram diferentes tipos de estômatos para espécies do mesmo gênero, no presente trabalho ocorreu o inverso, espécies de diferentes gêneros e famílias apresentaram o mesmo gênero. Com isso, estudos dessa natureza devem ser incentivados, ademais outras ferramentas devem ser incluídas para o melhor entendimento da funcionalidade, tipologia e implicações na evolução das espécies.

Tabela 2. Descrição da epiderme das dez espécies florestais amazônicas.

\begin{tabular}{|c|c|c|c|c|}
\hline Espécie & Tipo de Tricoma & $\begin{array}{l}\text { Parede } \\
\text { celular }\end{array}$ & Tipo de Estômato & Posição \\
\hline Carapa guianensis Aubl. & Ausente & Curva & Anomocítico & Hipoestomática \\
\hline Spondias mombin $\mathrm{L}$. & $\begin{array}{l}\text { Filiforme, unicelular com } \\
\text { espiculas e ocorrem } \\
\text { estrelado }\end{array}$ & Curva & Anomocítico & Anfiestomática \\
\hline Dipteryx odorata (Aubl.) Willd. & $\begin{array}{l}\text { Filiformes ramificados, } \\
\text { unicelulares com cerca de } 7 \\
\text { hastes }\end{array}$ & Sinuosa & Anomocítico & Hipoestomática \\
\hline $\begin{array}{l}\text { Lecythis lurida (miers) } \\
\text { S.A.Mori. }\end{array}$ & Ausente & Curva & Anomocítico & Hipoestomática \\
\hline $\begin{array}{l}\text { Vismia guianensis (Aubl.) } \\
\text { Choisy. }\end{array}$ & $\begin{array}{l}\text { Filiformes estrelado, } \\
\text { unicelulares com cerca de } 5 \\
\text { a } 6 \text { hastes }\end{array}$ & Sinuosa & Anomocítico & Hipoestomática \\
\hline Cenostigma tocantinum Ducke. & $\begin{array}{l}\text { Filiforme, unicelulares e } \\
\text { glandulares unicelulares }\end{array}$ & $\begin{array}{l}\text { Curva e } \\
\text { sinuosa }\end{array}$ & Anomocítico & Hipoestomática \\
\hline Alexa grandiflora Ducke. & $\begin{array}{l}\text { Filiforme, unicelular com } \\
\text { espiculas }\end{array}$ & Curva & Anomocítico & Hipoestomática \\
\hline Guazuma ulmifolia Lam. & $\begin{array}{l}\text { Filiforme estrelado e com } \\
\text { cerca de } 8 \text { a } 9 \text { hastes sendo } \\
\text { um central disposta a } 90^{\circ}\end{array}$ & Curva & Anomocítico & Hipoestomática \\
\hline $\begin{array}{l}\text { Dicypellium caryophyllaceum } \\
\text { (Mart.) Nees. }\end{array}$ & $\begin{array}{l}\text { Filiforme, unicelular com } \\
\text { espiculas }\end{array}$ & $\begin{array}{l}\text { Curva e } \\
\text { sinuosa }\end{array}$ & Anomocítico & Hipoestomática \\
\hline $\begin{array}{l}\text { Virola surinamensis (Rol. Ex } \\
\text { Rottb.) Warb. }\end{array}$ & $\begin{array}{l}\text { Filiforme estrelado e com } \\
\text { cerca de } 5 \text { a } 6 \text { hastes sendo } \\
\text { um central disposta a } 90^{\circ}\end{array}$ & Curva & Anomocítico & Hipoestomática \\
\hline
\end{tabular}

Fonte: Autores.

Em nove de dez espécies foram encontradas estômatos apenas na região abaxial, sendo assim classificadas como hipoestomáticas, resultados semelhantes foram descritos por Ferreira et al, (2015) em que, de onze espécies lenhosas do estado de Roraima, nove também foram classificadas por hipoestomáticas, podendo assim ser uma característica comuns as espécies ocorrentes na Amazônia. Entretanto, mais espécies devem ser analisadas no intuito de confirmar a hipótese, já que esse tipo folha são facilmente encontrados na natureza. Já a espécie Spondias mombin é anfiestomática, assim como encontrado em Spondias purpurea por Rocha et al. (2015).

Purkhust (1978), em sua teoria, relata que esse tipo de folhas (anfiestomáticas) são predominantes de plantas em ambientes secos, em virtude dos estômatos facilitam as trocas gasosas diminuindo a resistência da folha para a difusão dos gases. Através dos poros estomáticos o $\mathrm{CO} 2$ e o vapor de água atravessam a barreira imposta pela camada limítrofe e o tecido epidérmico, o que pode ocorrer em plantas de ambientes ensolarados, ou seja, com alta demanda evaporativa e alta irradiância.

Cinco tipos de tricomas foram encontrados (Figura 3), sendo eles: papiloso, estrelado, ramificado, glandular e 
filiforme. Spondias mombin, além de apresentar tricoma estrelado próximo da nervura central, ela foi a única a apresentar tricoma do tipo papiloso em toda a folha, porém em maior densidade na região da nervura central.

Foi possível observar o tricoma do tipo estrelado em mais três espécies sendo elas Vismia guianensis nas faces abaxial e adaxial, porém com maior concentração na abaxial, Virola surinamensis apenas na face abaxial e Guazuma ulmifolia em toda a folha em ambas as faces, sendo esta última contendo também o tricoma do tipo filiforme na área da nervura central na face abaxial. Duarte et al, (2011), também encontraram na espécie Guazuma ulmifolia tricomas do tipo estrelado, Rocha e Neves (2000) descrevem na espécie Hibiscus tiliaceus tricomas no mesmo formato, e Dardengo et al, (2017) encontraram na espécie Theobroma speciosum tricoma estrelado, sendo assim, este podendo ser uma característica da família.

O tricoma do tipo filiforme também foi encontrado em outras três espécies da família fabaceae, caracterizando, portanto, como característica da família, sendo estas espécies Alexa grandiflora em ambas as faces, Dicypellium caryophyllaceum na face abaxial e Cenostigma tocantinum próximo da nervura central sendo, nesta última espécie, encontrado também tricoma do tipo glandular em toda a folha. A Dipteryx odorata foi a única espécie onde encontrou-se tricoma do tipo ramificado próximo da nervura central. Já nas espécies Carapa guianensis e Lecythis lurida, não foram encontrados nenhum tipo de tricoma.

Ressalta-se que todas as espécies foram coletadas em floresta tropical úmida fechada e são espécies que chegam no dossel floresta e Johnson (1975) explica que os tricomas aumentam a transpiração por constituírem superfície adicional no processo de evaporação, sendo este o motivo de nem todas as espécies apresentarem tricomas, já que as espécies vivem em ambiente de bastante incidência solar e necessitam de estratégias para diminuir a perda de água. 
Figure 3. A-K. Tipos de Tricomas encontrados: A. Tricoma papilooso Spondias mombin; B. Tricoma estrelado Spondias mombin; C. Tricoma ramificado Dipteryx odorata; D. Tricoma estrelado Vismia guianensis; E. Tricoma filiforme Cenostigma tocantinum; F. Tricoma glandular Cenostigma tocantinum; G. Tricoma filiforme Alexa grandiflora; H. Tricoma estrelado Guazuma ulmifolia; I. Tricoma aliforme Guazuma ulmifolia; J. Tricoma filiforme Dicypellium caryophyllaceum; K. Tricoma estrelado Virola surinamensis.

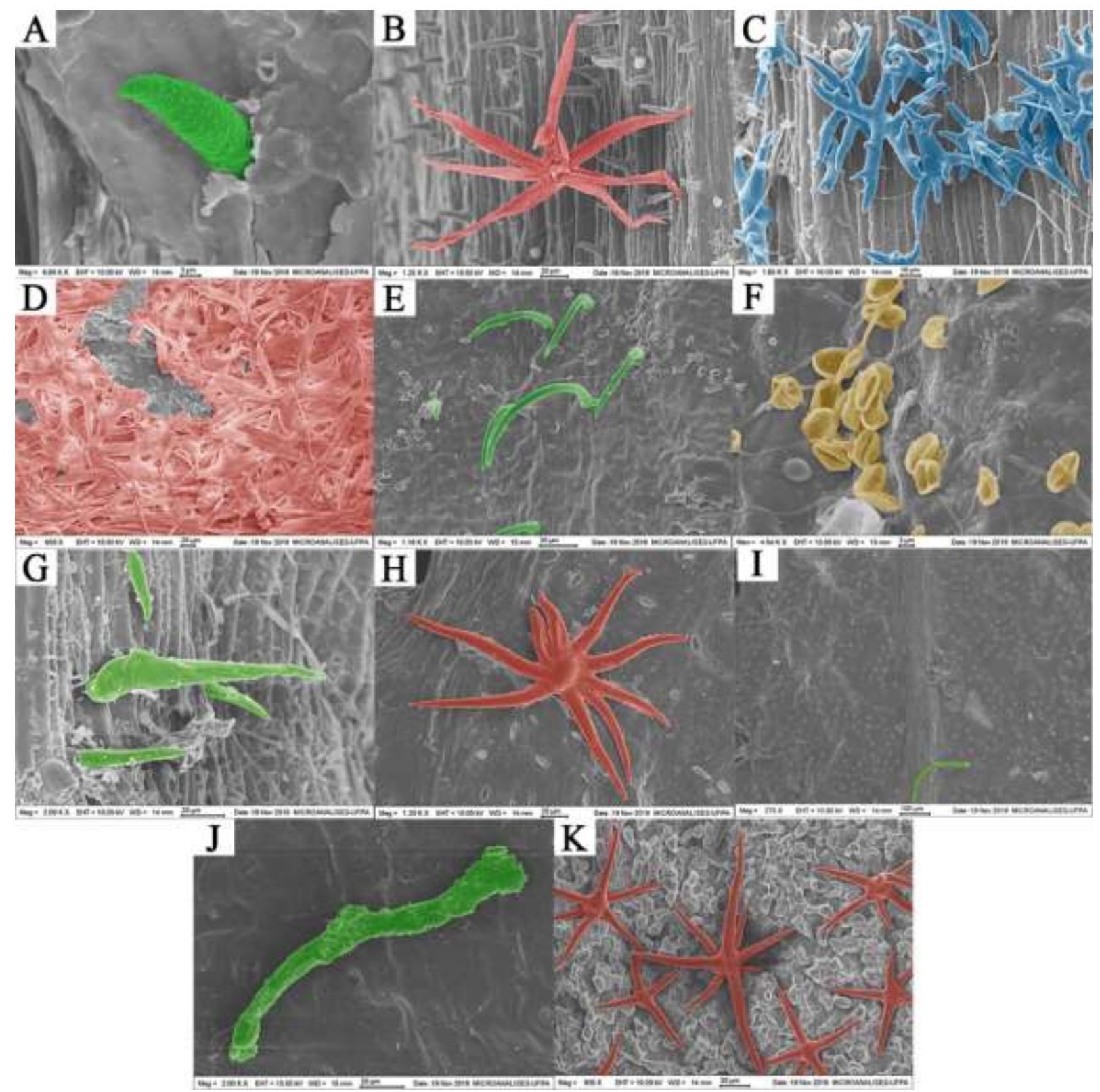

Fonte: Autores.

Na superfície epidérmica de cinco espécies foram encontrados depósitos (Figura 4), os quais submetidos a análises de EDS que identificou, em todas as espécies, a presença de carbono (C) e oxigênio (O). Na espécie Dipteryx odorata, o depósito foi detectado na face abaxial, este com o formato de esfera sendo identificados o elemento potássio (K), enquanto na espécie Vismia guianensis foram localizados tanto na região abaxial quanto na adaxial da folha, no formato de esfera e identificados os seguintes os elementos químicos sílica $(\mathrm{Si})$, alumínio $(\mathrm{Al})$, titânio $(\mathrm{Ti})$ e ferro $(\mathrm{Fe})$. Em Lecythis lurida o deposito possui formato de esfera com ornamentações, semelhando-se a coroa. Alquini et al (2003) citam que na superfície da cutícula pode haver cristais, borrachas, resinas e óleos e Corrêa et al (2008) enfatizam que esses podem estão relacionados a defesa da planta 
a herbivoria e ocorrem em plantas ricas em metabolitos secundários. Ressalta-se que esses elementos ocorrem de forma natural uma vez que a coleta foi realizada em ambiente com pouca e/ou nenhuma ação humana.

Algumas dessas espécies já estão sendo usadas como bioinseticidas naturais como realizado por Anjos et al. (2019) que utilizaram em seu experimento folhas secas de Vismia guianensis como alternativa de fungicida no manejo sustentável do nematoide-das-galhas do cafeeiro.

Figure 4. A-K. Depósitos encontrados na Microscopia Eletronica de Varredura e analise de EDS: A. Dipteryx odorata Abaxial; B. Lecythis lúrida Abaxial; C. Vismia guianensis Abaxial; D. Vismia guianensis Adaxial; E. Alexa grandiflora Nervura Central.
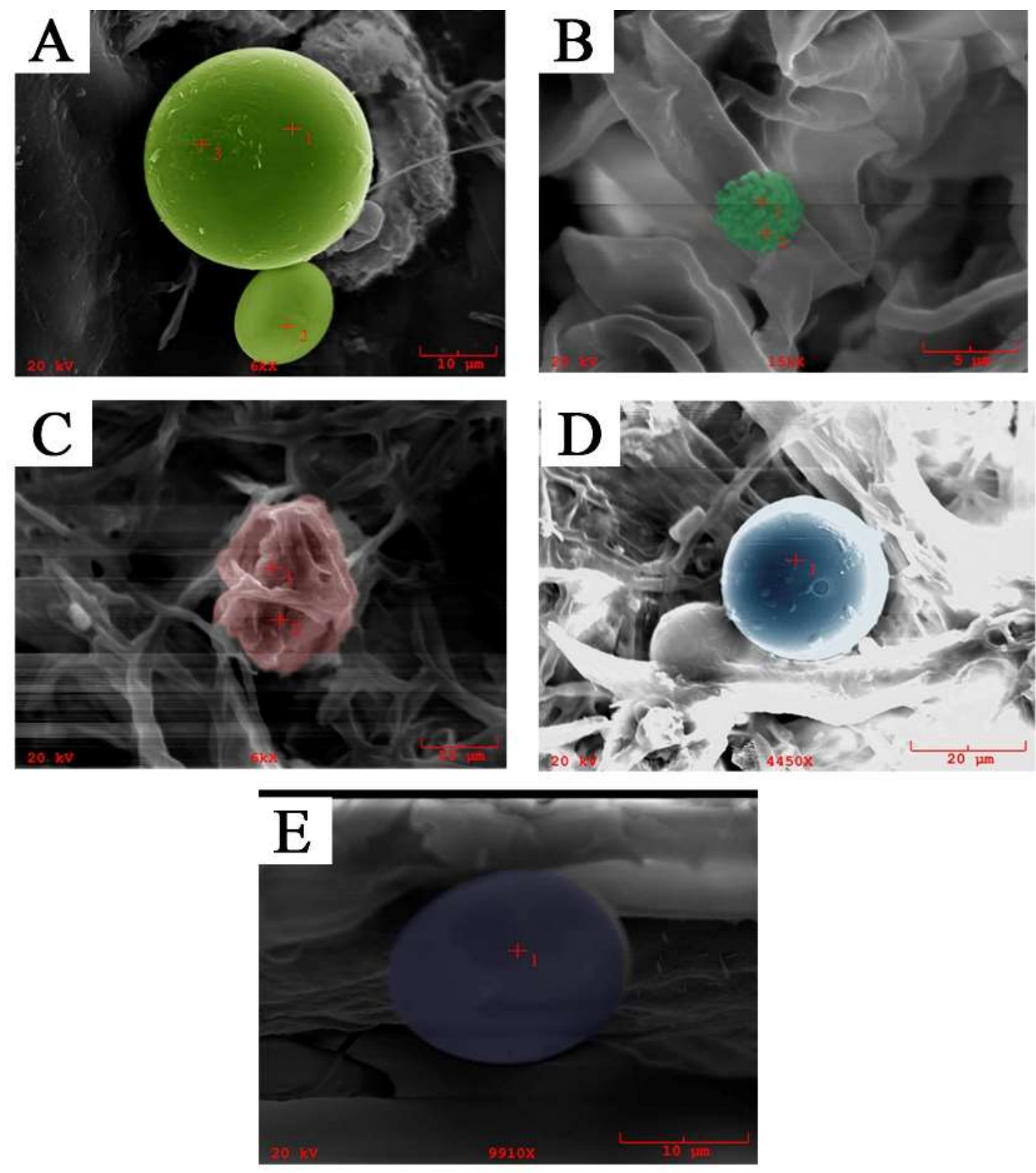

Fonte: Autores.

Foram encontradas ornamentações, na cera epicuticular que revestem a célula guarda em estômatos da espécie 
Dipteryx odorata e em toda região abaxial da espécie Virola surinamensis. De acordo com Metcalfe e Chalk (1979) e Barthlott et al (1998) a cera é classificada em placas orientadas verticalmente, formando um ângulos de $0^{\circ}$ a $90^{\circ}$. Os autores também citam a importância dessa estrutura na distinção e classificação dos vegetais. Assim como Arroyo e Cutler (1984), que encontraram que os diferentes padrões de ornamentação da cera epicuticular, explicam que esses padrões permitem a separação de espécies de diferentes gêneros da família, contribuindo assim para a particularização das espécies.

Além das ornamentações (Figura 5), encontrou-se epiderme papilosa na espécie Lecythis lurida na epiderme adaxial e na espécie Dicypellium caryophyllaceum na face adaxial. Silva et al. (2016), por meio das imagens obtidas por MEV também observou epiderme papilosa para o folíolo de Macrolobium longipes na face abaxial e os autores Oliveira et al. (2016) observam nas espécies Aechmea distichantha Lem. e Canistropsis billbergioides (Schult. \& Schult.f) Leme as células papilosas ocorrem apenas na face adaxial, Neinhuis e Barthlott (1977) destacam que a presença de epiderme papilosa torna a superfície foliar antiaderente e livre de contaminantes.

Figure 5. A-J. A,B. Ornamentação da cera epicuticular nos estômatos de Dipteryx odorata; B,C. Epiderme papilosa de Lecythis lúrida; E. Epiderme papilosa de Dicypellium caryophyllaceum; F. Ornamentação de Virola surinamensis.

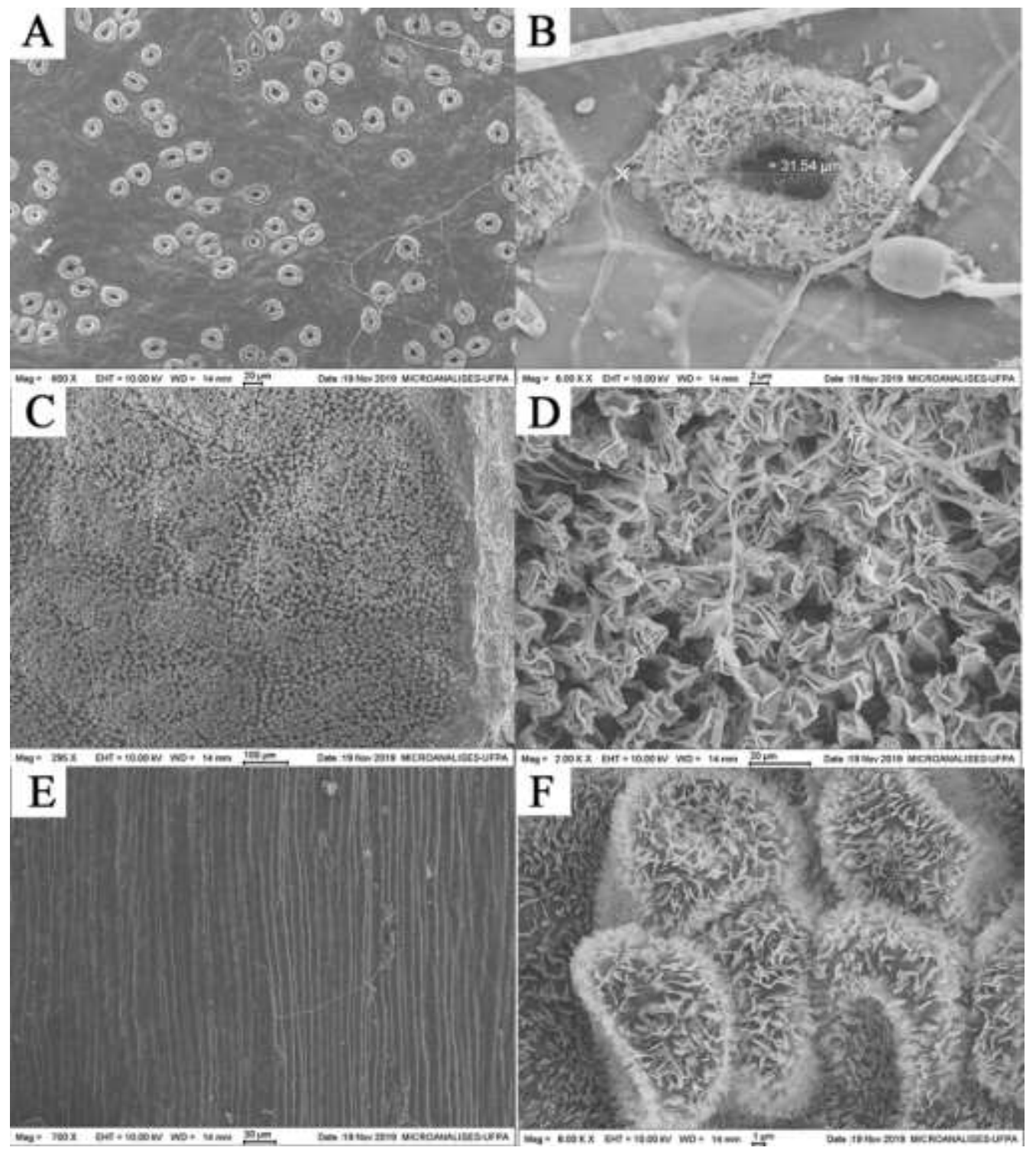

Fonte: Autores. 
A partir da mensuração com comprimento, largura e frequência estomática foram feitos gráficos de Box-plot (Figura 6), análise ANOVA e realizou-se a comparação entre as espécies e a avalição da simetria e dispersão dos dados, observandose, portanto, que a espécie Dipteryx odorata apresentou maior variação de dados se tratando do comprimento e largura dos estômatos, porém, nos dados de quantidade de estômatos.

Figure 6. Gráfico de Box-plot da ANOVA para comparação das espécies.

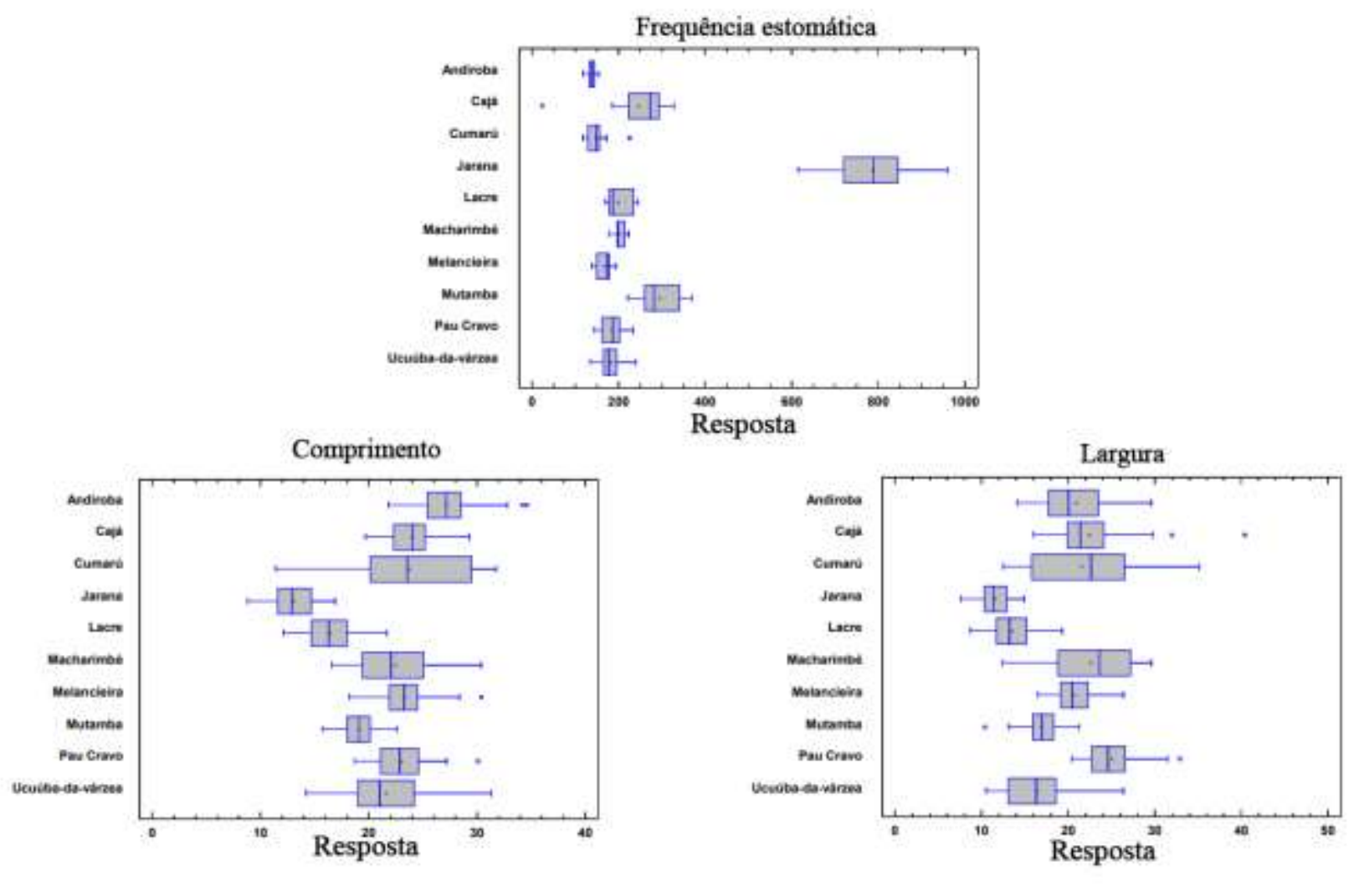

Fonte: Autores.

A espécie que apresentou maior variação foi a Lecythis lurida e as menores dispersões foram encontradas na espécie Cenostigma tocantinum na quantidade de estômatos, a Guazuma ulmifolia no comprimento dos estômatos e Alexa grandiflora para largura dos estômatos. Esses resultados também podem ser observados na tabela de médias e desvio padrão (Tabela 3), que demonstram numericamente os resultados apresentados pelos gráficos Box-plot.

Metcalfe e chalk $(1950,1979)$ relatam sobre a importância, benefícios e dificuldade para se trabalhar com dados quantitativos do limbo foliar, em virtude da plasticidade da folha. Contudo, esses autores afirmam também que esse caractere pode evidenciar particularidades das espécies. Com o avanço das ferramentas matemáticas e estatísticas, esse caractere tem se tornado cada vez mais preciso, com isso alguns trabalhos já afirmam que é possível separar as espécies com caracteres quantitativos conforme trabalhos realizados por Shaheen et al (2020), Rashid et al (2018) e Klimko et al (2018) 
Tabela 3. Média e desvio padrão das espécies obtidos através da ANOVA.

\begin{tabular}{|c|c|c|c|}
\hline Espécie & $\begin{array}{l}\text { Frequência } \\
\text { estomática }\end{array}$ & $\begin{array}{l}\text { Comprimento dos estômatos } \\
(\mu \mathrm{m})\end{array}$ & $\begin{array}{c}\text { Largura dos } \\
\text { estômatos }(\mu \mathrm{m})\end{array}$ \\
\hline Carapa guianensis Aubl. & $138,60 \pm 10,91$ & $27,19 \pm 2,72$ & $20,90 \pm 3,95$ \\
\hline Spondias mombin $\mathrm{L}$. & $246,55 \pm 89,34$ & $24,00 \pm 2,49$ & $22,45 \pm 4,14$ \\
\hline Dipteryx odorata (Aubl.) Willd. & $150,3 \pm 31,33$ & $23,76 \pm 5,74$ & $21,58 \pm 5,98$ \\
\hline Lecythis lurida (miers) S.A.Mori. & $787,5 \pm 103,34$ & $13,04 \pm 1,97$ & $11,54 \pm 1,77$ \\
\hline Vismia guianensis (Aubl.) Choisy. & $198,9 \pm 29,60$ & $16,42 \pm 2,27$ & $13,55 \pm 2,26$ \\
\hline Cenostigma tocantinum Ducke. & $200,9 \pm 15,19$ & $22,48 \pm 3,43$ & $22,62 \pm 4,91$ \\
\hline Alexa grandiflora Ducke. & $168,30 \pm 18,08$ & $23,274 \pm 2,34$ & $20,68 \pm 2,32$ \\
\hline Guazuma ulmifolia Lam. & $294,30 \pm 52,38$ & $19,08 \pm 1,49$ & $16,91 \pm 2,02$ \\
\hline Dicypellium caryophyllaceum (Mart.) Nees. & $184,6 \pm 26,89$ & $23,00 \pm 2,37$ & $25,01 \pm 3,07$ \\
\hline Virola surinamensis (Rol. Ex Rottb.) Warb. & $182,50 \pm 29,55$ & $21,548 \pm 3,72$ & $16,43 \pm 3,62$ \\
\hline
\end{tabular}

Fonte: Autores.

Através das análises do PCA da contagem de estômatos, comprimento e largura, foi possível agrupar as espécies Alexa grandiflora e Lecythis lurida no mesmo quadrante (Figura 7) nos três gráficos, apesar de não pertencerem a mesma família e nem semelhanças morfologicas. Isso revela a similaridade no tamanho e frequência dos estômatos nas espécies estudadas. Morel et al (2021) também utilizaram análises multivariada para distinção de espécies do gênero Xyris e obtiveram sucesso no uso da ferramenta.

Figure 7. Análise de componentes principais das dez espécies arbóreas levando em consideração a quantidade de estômatos, comprimento e largura.

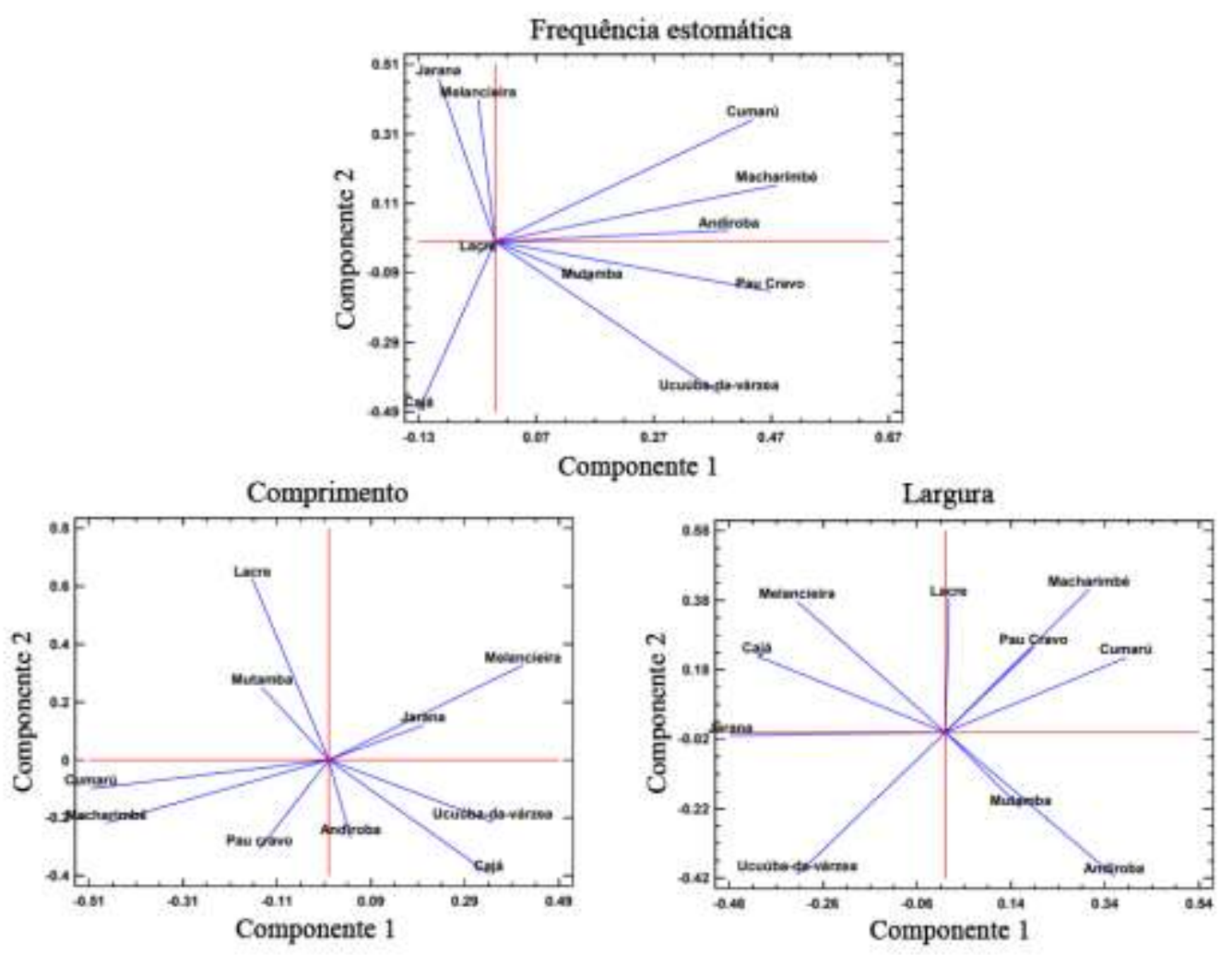

Fonte: Autores. 
$\mathrm{Na}$ análise de cluster (Figura 8), ou análise de agrupamento, foi possível realizar o agrupamento por similaridade das espécies onde, na quantidade de estômatos, as espécies foram divididas em três cluster, sendo o primeiro cluster com oito espécies, o segundo apenas com a espécie Dipteryx odorata e o terceiro cluster apenas com a espécie Cenostigma tocantinum. Esse resultado demonstra que, as espécies pertencentes aos cluster dois e três são facilmente identificadas através da análise de cluster a partir da contagem dos estômatos, já que elas não foram agrupadas com nenhuma outra espécie. Nos gráficos de comprimento a largura, os agrupamentos gerados não foram satisfatórios, fazendo com que a análise de cluster para comprimento e largura não seja eficiente.

Figure 8. Gráficos de agrupamento das análises de cluster das espécies.
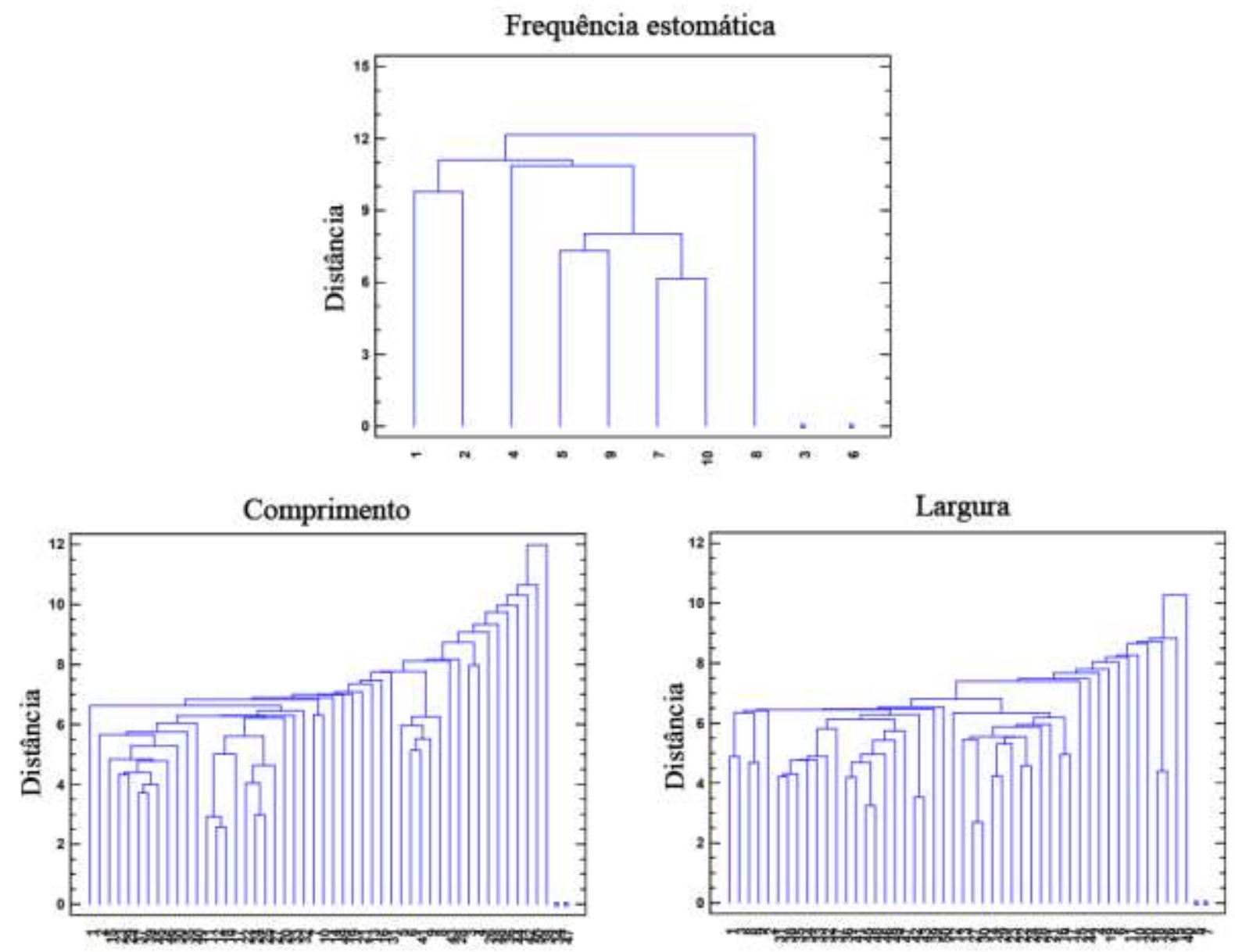

Fonte: Autores.

\section{Conclusão}

As espécies de Fabaceae são as que possuem maior diversidade de estrutura dentro da família o que dificulta o agrupamento delas;

A forma dos tricomas podem ser utilizados com sucesso para distinção de espécies.

A frequência estomática pode ser utilizado com sucesso para agrupamento das espécies estudadas;

As espécies Dipteryx odorata e Virola surinamensis podem ser facilmente identificadas devido o tipo de cera epicuticular encontrada em sua célula guarda.

As espécies Alexa grandiflora e Lecythis lurida apesar de serem de famílias botânicas diferentes, apresentam 
estruturas semelhantes;

Somente a espécie Spondias mombin pode ser identificada, quanto a posição dos estômatos, por ser anfiestomática. Análise de agrupamento da frequência estomática é a que melhor distingue os agrupamentos.

\section{Agradecimentos}

Os autores agradecem ao CNPq; FAPESPA (ICAAF 2018) pelo apoio no financiamento.

\section{Referências}

Alquini, Y., Bona, C., Boeger, M. R. T., Costa, C. G. da, \& Barros, C. F. (2006). Epiderme. In B. Appezzato-da-Glória \& S. M. Carmello-Guerreiro (Eds.), Anatomia vegetal (2a). Viçosa-MG.

Anjos, E. F. M. dos, Vieira Júnior, J. R., Fernandes, C. D. F., Rocha, R. B., Santos, C. F., Ramos, N. P., \& Silva, G. R. da. (2019). Biofumigação de substratos com espécies amazônicas para produção de mudas de cafeeiro livres do nematoide-das-galhas. Revista Ibero-Americana de Ciências Ambientais, 10(2), 25-34. https://doi.org/10.6008/cbpc2179-6858.2019.002.0003.

Araújo, M. G. P. de, \& Mendonça, M. S. de. (1998). Escleromorfismo foliar de Aldina heterophylla Spruce ex Benth. (Leguminosae: Papilionoideae) em três campinas da Amazônia Central. Acta Amazônica, 28(4), 353-371. Retrieved from https://doi.org/10.1590/1809-43921998284371.

Arroyo, S. C., \& Cutler, D. F. (1984). Evolutionary and taxonomic aspects of the internal morphology in Amaryllidaceae from South America and Southern Africa. Kew Bulletin, 39(3), 467-498. Retrieved from https://doi.org/10.2307/4108592.

Barthlott, W., Neinhuis, C., Cutler, D., Ditsch, F., Meusel, I., Theisen, I., \& Wilhelmi, H. (1998). Classification and terminology of plant epicuticular waxes. Botanical Journal of the Linnean Society, 126(3), 237-260. https://doi.org/10.1006/boj1.1997.0137.

Carpenter, K. J. (2006). Specialized structures in the leaf epidermis of basal angiosperms: Morphology, distribution, and homology. American Journal of Botany, 93(5), 665-681. https://doi.org/10.3732/ajb.93.5.665.

Chen, J. H., Sun, H., \& Yang, Y. P. (2008). Comparative morphology of leaf epidermis of Salix (Salicaceae) with special emphasis on sections Lindleyanae and Retusae. Botanical Journal of the Linnean Society, 157(2), 311-322. https://doi.org/10.1111/j.1095-8339.2008.00809.x.

Corrêa, P. G., Pimentel, R. M. de M., Cortez, J. S. de A., \& Xavier, H. S. (2008). Herbivoria e anatomia foliar em plantas tropicais brasileiras. Ciênc. Cult. (Säo Paulo), 60(3), 54-57.

Cysneiros, V. C., Mendonça Júnior, J. O., Lanza, T. R., Moraes, J. C. R., \& Samor, O. J. M. (2018). Espécies madeireiras da Amazônia: riqueza, nomes populares e suas peculiaridades. Pesquisa Florestal Brasileira, 38. https://doi.org/10.4336/2018.pfb.38e201801567.

Dardengo, Juliana de Freitas Encinas; Rossi, Ana Aparecida Bandini; Silva, Ivone Vieira da; Pessoa, Marcos José Gomes; Silva, C. J. da S. (2017). Análise da inlfuência luminosa nos aspectos anatômicos de folhas de Theobroma speciosum WILLD EX SPRENG. (Malvaceae). Ciência Florestal, 27(3), 843-851. Retrieved from https://www.scielo.br/scielo.php?script=sci_arttext\&pid=S1980-50982017000300843.

Deng, M., Hipp, A., Song, Y. G., Li, Q. S., Coombes, A., \& Cotton, A. (2014). Leaf epidermal features of Quercus subgenus Cyclobalanopsis (Fagaceae) and their systematic significance. Botanical Journal of the Linnean Society, 176(2), 224-259. https://doi.org/10.1111/boj.12207.

Duarte, M. R., Dranka, E. R. K., \& Yano, M. (2011). Análise Microscópica Foliar De Mutamba (Guazuma ulmifolia LAM., MALVACEAE). Visão Acadêmica, 12(2), 7-17. Retrieved from https://revistas.ufpr.br/academica/article/view/27252/18154.

Ergen AkçIn, Ö., Şenel, G., \& AkçIn, Y. (2013). Leaf epidermis morphology of some Onosma (Boraginaceae) species from Turkey. Turkish Journal of Botany, 37(1), 55-64. https://doi.org/10.3906/bot-1202-33.

Ferreira, C. S., do Carmo, W. S., Graciano-Ribeiro, D., de Oliveira, J. M. F., de Melo, R. B., \& Franco, A. C. (2015). Anatomia da lâmina foliar de onze Espécies lenhosas dominantes nas savanas de Roraima. Acta Amazonica, 45(4), 337-346. https://doi.org/10.1590/1809-4392201500363.

Gul, S., Ahmad, M., Zafar, M., Bahadur, S., Celep, F., Sultana, S., ... Ayaz, A. (2019a). Taxonomic significance of foliar epidermal morphology in Lamiaceae from Pakistan. Microscopy Research and Technique, 82(9), 1507-1528. https://doi.org/10.1002/jemt.23316.

Gul, S., Ahmad, M., Zafar, M., Bahadur, S., Sultana, S., Ashfaq, S., \& Siddiq, Z. (2019b). Foliar epidermal anatomy of Lamiaceae with special emphasis on their trichomes diversity using scanning electron microscopy. Microscopy Research and Technique, 82(3), 206-223. https://doi.org/10.1002/jemt.23157.

Johnson, H. B. (1975). Plant pubescence: An Ecological Perspective. The Botanical Review, 41(3), 233-258. Retrieved from https://link.springer.com/article/10.1007/BF02860838.

Junqueira, C. U. (1990). O uso de cortes finos de tecido na medicina e biologia. Meios \& Métodos, 66, 10-11.

Klimko, M., Nowińska, R., Wilkin, P., \& Wiland-Szymańska, J. (2018). Comparative leaf micromorphology and anatomy of the dragon tree group of Dracaena (Asparagaceae) and their taxonomic implications. Plant Systematics and Evolution, 304(8), 1041-1055. https://doi.org/10.1007/s00606-018-1530-3.

Kong, H. Z. (2001). Comparative morphology of leaf epidermis in the Chloranthaceae. Botanical Journal of the Linnean Society, 136(3), 279-294. https://doi.org/10.1006/boj1.2001.0442. 
Research, Society and Development, v. 10, n. 2, e58810212961, 2021

(CC BY 4.0) | ISSN 2525-3409 | DOI: http://dx.doi.org/10.33448/rsd-v10i2.12961

Kraus, J. E., \& Arduin, M. (1997). Manual básico de métodos em morfologia vegetal. Seropédica.

Mele, G., \& Gargiulo, L. (2020). Automatic cell identification and counting of leaf epidermis for plant phenotyping. MethodsX, 7. https://doi.org/10.1016/j.mex.2020.100860.

Metcalfe, C. R., \& Chalk, L. (1950). Anatomy of the dicotyledons (Oxford, Ed.).

Metcalfe, C. R., \& Chalk, L. (1979). Anatomy of the dicotyledons (Oxford, Ed.).

Morel, J., Moguédec, G. LE, \& Munzinger, J. (2021). Multivariate morphometric analysis supported by an anatomical approach to assess species delimitation in Xyris (Xyridaceae) in New Caledonia. Botanical Journal of the Linnean Society, 195(XX-XX), 1-14. https://doi.org/10.1093/botlinnean/boaa108.

Nazish, M., Ahmad, M., Ullah, R., Shahat, A. A., Potter, D., Zafar, M., \& Sultana, S. (2020). Taxonomic implications of leaf epidermis in halophytes of Amaranthaceae from Salt Range of Punjab, Pakistan. Plant Biosystems. https://doi.org/10.1080/11263504.2020.1837277.

Oliveira, F. M. C. de, Souza, A. M. de, Corrêa, B. B. R., Maeda, T. M., \& Melo-de-Pinna, G. F. (2016). Anatomia floral de Aechmea distichantha Lem. e Canistropsis billbergioides (Schult. \& Schult.f) Leme (Bromeliaceae). Hoehnea, 43(2), 183-193. https://doi.org/10.1590/2236-8906-78/2015.

Parkhurst, D. F. (1978). The Adaptive Significance of Stomatal Occurrence on One or Both Surfaces of Leaves. The Journal of Ecology, 66(2), 367. https://doi.org/10.2307/2259142.

Rashid, N., Zafar, M., Ahmad, M., Khan, M. A., Malik, K., Sultana, S., \& Shah, S. N. (2019). Taxonomic significance of leaf epidermis in tribe Trifolieae L. (Leguminosae; Papilionoideae) in Pakistan. Plant Biosystems, Vol. 153, pp. 406-416. https://doi.org/10.1080/11263504.2018.1492995.

Rêgo, L. J. S., Silva, M. L., Silva, L. F., Gama, J. R. V., \& Reis, L. P. (2016). Comercialização da amêndoa de cumarunos municípios de Santarém e Alenquer, leste da Amazônia. Revista de Administração e Negócios da Amazônia, 8(3), 338-361. https://doi.org/10.18361/2176-8366/rara.v8n3p338-361.

Rocha, L. A., Rocha, A. M., Pacheco, A. C. L., \& Abreu, M. C. de. (2015). Diferenças foliares morfoanatômicas de quatro espécies da família Anacardiaceae. Caderno de Pesquisa. Série Biologia, 27(2), 35-48. Retrieved from http://dx.doi.org/10.17058/cp.v27i2.6798\%0A.

Shaheen, S., Fateh, R., Younis, S., Harun, N., Jaffer, M., Hussain, K., \& Khan, F. (2020). Light and scanning electron microscopic characterization of thirty endemic Fabaceae species of district Lahore, Pakistan. Microscopy Research and Technique, 83(12), 1507-1529. https://doi.org/10.1002/jemt.23545.

Silva, N. R. da, Oliveira, M. W. da S., Almeida Filho, H. A. de, Pinheiro, L. F. S., Rossatto, D. R., Kolb, R. M., \& Bruno, O. M. (2016). Leaf epidermis images for robust identification of plants. Scientific Reports, 6, 1-10. https://doi.org/10.1038/srep25994.

Sousa, L. B. (2018). Análise de cluster para avaliação da divergência genética em variedades de mangaba. Universidade Federal de Uberlânsia.

Tomaszewski, D., Byalt, A., \& Gawlak, M. (2019). Leaf and stem epicuticular wax structures in Lonicera species (Caprifoliaceae). Nordic Journal of Botany, 37(5), 1-9. https://doi.org/10.1111/njb.02210.

Vicini, L. (2005). Análise multivariada da teoria à prática. UFSM. 215p. 